\section{ABCS}

How to cite this article: Crespo et al Dexamethasone intravitreal implant for macular edema: a pilot study in a public health center. ABCS Health Sci. 2020;45(Suppl.3):e020104. https://doi. org/10.7322/abcshs.2020S04.1851

Corresponding Author: Vagner Loduca Lima - Discipline of Ophthalmology, Centro Universitário FMABC - Avenida Lauro Gomes, 2000 - Sacadura Cabral - CEP 09060-650 - Santo André (SP), Brazil -

Email:vagner@loduca.com.br

Declaration of interests: nothing to declare

This is an open access article distributed under the terms of the Creative Commons Attribution License

(C) 2020 Crespo et al.

\title{
Dexamethasone intravitreal implant for macular edema: a pilot study in a public health center
}

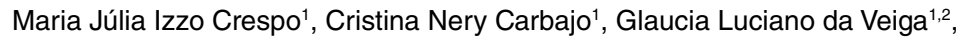
Fernando Luiz Affonso Fonseca ${ }^{1,2,3}$, Vagner Loduca Lima ${ }^{1}$, Júlio Zaki Abucham Neto ${ }^{1}$

${ }^{1}$ Discipline of Ophthalmology, Centro Universitário FMABC - Santo André (SP), Brazil. ${ }^{2}$ Clinical Analysis Laboratory, Centro Universitário FMABC - Santo André (SP), Brazil.

${ }^{3}$ Department of Pharmaceutical Sciences, Universidade Federal de São Paulo (UNIFESP) - Diadema (SP), Brazil.
\end{abstract}

\section{ABSTRACT}

Introduction: Macula edema consists in one of the most common causes of visual impairment. Objective: To evaluate the safety and efficacy of continuous release of $0.7 \mathrm{mg}$ dexamethasone (using implantable device) for treatment of macular edema. Methods: Cross-sectional observational study of 16 patients treated with $0.7 \mathrm{mg}$ dexamethasone intraocular implant. Visual acuity, intraocular pressure and central macular thickness were recorded at baseline, 1-month and 3-month follow-up. Results: 15 eyes of 13 patients were included. Most eyes $(n=9)$ improve visual acuity from baseline at 1-month follow-up; this improvement persisted through 3-monyh follow-up in six eyes. The central macular thickness decreased in the majority of the subjects at 1 -month $(n=12)$ and at 3 -month $(n=10)$ follow-up. Three eyes presented with elevated intraocular pressure. Conclusion: Dexamethasone implant can both reduce the risk of vision loss and improve anatomical features of macular edema due to several pathologies studied. This implant may be used safely and should be considered a therapeutic option to Brazilian Public Health System.

Keywords: intravitreal dexamethasone implant; macular edema; anti-vascular endothelial growth factor; diabetes.

\section{INTRODUCTION}

Macula edema (ME) consists in one of the most common causes of visual impairment in patients with several ocular pathologies such as diabetic retinopathy, wet age-related macular degeneration, central retina vein occlusion, uveitis, among others. Vascular endothelial growth factor (VEGF) is responsible not only for the increase of abnormal vascular permeability but also for stimulating angiogenesis, playing a crucial role in the pathophysiology of $\mathrm{ME}^{1,2}$. In the past couple decades, intravitreal injections of antiVEGF agents have shown efficacy in the treatment of ME and, nowadays, are recommended as first line therapy for macula edema management, especially diabetic macular edema $^{3,4}$. Three intravitreal VEGF inhibitors have been shown to be beneficial and safe for this purpose: ranibizumab, bevacizumab and aflibercept.

However, some patients do not present with substantial improvement in visual acuity, despite the treatment with several intravitreal injections of anti-VEGF agents. Considering that ME could also be associated with an inflammatory pathological 
process, intravitreal steroids have been used as alternative drugs in selected cases. Intravitreal injections of triamcinolone have been effective in reduction of ME in this scenario, but often several injections are required increasing the occurrence of its adverse effects, such as cataract formation, increased intraocular pressure (IOP) and steroid-induced glaucoma. Advances in technology have led to the development of intravitreal implants that can gradually release corticosteroids directly in the back of the eye. Most recently, a bioerodible, intravitreal, sustained-released implant has been developed that delivers dexamethasone up to six months to the posterior chamber (DEX implant Ozurdex; Allergan, Inc, Irvine, California) $)^{5,6}$.

Several studies have already shown the morphological improvement and visual acuity gain as predictable outcomes of these treatment alternative, yet the intravitreal implant of dexamethasone is not widely available in Brazilian public health system (SUS).

In this study we aim to evaluate the outcomes of continuous release of $0,7 \mathrm{mg}$ dexamethasone (using implantable device) in patients with ME attended in the Ophthalmology Department at the Centro Universitário FMABC in 2019, Santo Andre - Brazil.

\section{METHODS}

This cross-sectional observational study was conducted in compliance with the Declaration of Helsinki and was approved by the local institutional review board.

We reviewed the medical records of 16 patients enrolled in the "Hands on" program sponsored by Allergan in 2019. This program aimed to train retina fellowship ophthalmologists how to master the intravitreal implants injections technique. Therefore, Allergan donated 16 intravitreal dexamethasone implants used in this study (DEX implant Ozurdex; Allergan, Inc, Irvine, California) without any costs to the patients.

Inclusion criteria were as follows: 1) any etiology of ME; 2) eyes previously treated with anti-VEGF agents or naïve of treatment. Key exclusion criteria included the following: 1) any active ocular infection; 2) history of major ocular surgery (vitrectomy); 3) aphakia; 4) history of glaucoma.

Data collected were standardized and included demographics, ME etiology, best corrected visual acuity (BCVA) using Snellen charts (converted into decimal notation), slit-lamp biomicroscopy examination, IOP measurement via applanation tonometry, dilated fundus examination and optical coherence tomography (OCT) with central macular thickness measurement (OCT Spectralis ${ }^{\oplus}$; Heidelberg Engineering, Heidelberg, Germany). Each patient was examined prior to the injection and follow-up at 01 week, 01 and 03 months after the procedure. In every visit a full ophthalmic examination as described was performed.

All the individuals selected to this study underwent intravitreal injection of the dexamethasone $(0,7 \mathrm{mg})$ implant into the study eye following all aseptic precautions in a sterile environment. Prior to the injection the eye was anesthetized with topical and subconjunctival anesthetics and prepared with topic iodine solution. The implant was injected in the inferotemporal quadrant of the study eye. All patients were treated with a topic ophthalmic antibiotic 4 times a day for 01 week after the injection and headed to the Ophthalmological Emergency Room if any alert sign.

All collected data are resumed on the following tables and images.

\section{RESULTS}

Sixteen patients received intravitreal dexamethasone implants on the training program "Hands on" sponsored by Allergan in 2019. However, three patients were excluded from this analysis duo to lack of information on the medical records. Out of the 13 eligible participants, 15 eyes received the intravitreal implant and were individually analyzed.

Patient demographics and baseline characteristics are listed in Table 1 . There was a total of 7 men and 6 women. The most prevalent cause of ME was diabetes mellitus (10 subjects), follow by uveitis ( 2 subjects) and central retina vein occlusion (1 subject). The underlying causes of the uveitis were: Vogt Koynagi Harada and sarcoidosis.

The mean number of previous intravitreal injections administered was 7 (range from 0 to 18) (Table 1). Regarding the antiVEGF agents currently available for intravitreal injections, there was a total amount of 77 bevacizumab, 15 triamcinolone, six combined bevacizumab + triamcinolone, six aflibercept and five ranibizumab.

The BCVA range from 0.01 to 0.8 among the 15 eyes. We considered visual acuity improvement any numerical gain. At 1-month follow-up after the dexamethasone implant was placed, nine subjects experienced visual acuity improvement and two subjects remained stable. However, four patients showed worsening of BCVA. At 3-month follow-up, six patients revealed BCVA improvement, on the other hand the same number of patients demonstrated BCVA worsening. BCVA of three subjects remained stable (Table 2).

Out of the 15 studied eyes, three presented with elevated IOP (Table 3). Two eyes of the same patient revealed IOP of $28 \mathrm{mmHg}$ measured via applanation tonometry at 1-week and 1-month follow-up visits. We were able to manage his IOP with timolol maleate $0.5 \%$ and brimonidine tartrate $0.2 \%$. The third case of elevated IOP presented with a $4 \mathrm{mmHg}$ increase in both 1-month and 3-month follow-up. This patient was already using hypotensive eye drops thus, the prescription has not been changed. No additional treatment with surgery or laser was necessary in our patients.

Regarding the central macular thickness, one subject did not meet the inclusion criteria for OCT imaging protocol due to 
Table 1: Diagnosis, number and type of previous applications.

\begin{tabular}{|c|c|c|c|c|c|c|c|}
\hline \multirow[b]{2}{*}{ Patients } & \multirow[b]{2}{*}{ Diagnosis } & \multirow{2}{*}{$\begin{array}{l}\text { Number of } \\
\text { Application }\end{array}$} & \multicolumn{5}{|c|}{ Type of previous applications } \\
\hline & & & Bevacizumab & $\begin{array}{l}\text { Bevacizumab } \\
\text { Triancinolona }\end{array}$ & Triancinolona & Ranibizumab & Aflibercept \\
\hline 1 & \multirow{7}{*}{ CME } & 18 & 14 & 1 & 0 & 0 & 3 \\
\hline 2 & & 8 & 7 & 0 & 0 & 0 & 1 \\
\hline 3 & & 17 & 81 & 2 & 3 & 3 & 1 \\
\hline 4 & & 8 & 3 & 2 & 2 & 0 & 1 \\
\hline 5 & & 10 & 6 & 0 & 4 & 0 & 0 \\
\hline 6 & & 5 & 5 & 0 & 0 & 0 & 0 \\
\hline 7 & & 5 & 4 & 1 & 0 & 0 & 0 \\
\hline 8 & VKH & 1 & 0 & 0 & 1 & 0 & 0 \\
\hline 9 & DME & 7 & 6 & 0 & 1 & 0 & 0 \\
\hline 10 & CRVO & 6 & 5 & 0 & 1 & 0 & 0 \\
\hline 11 & \multirow{2}{*}{ DME } & 6 & 6 & 0 & 0 & 0 & 0 \\
\hline 12 & & 7 & 5 & 0 & 0 & 2 & 0 \\
\hline 13 & SO & 0 & 0 & 0 & 0 & 0 & 0 \\
\hline 14 & \multirow{2}{*}{ DME } & 6 & 4 & 0 & 2 & 0 & 0 \\
\hline 15 & & 5 & 4 & 0 & 1 & 0 & 0 \\
\hline
\end{tabular}

CME: cystoid macular edema; CRVO: central retinal vein occlusion. DME: Diabetic macular edema; SO: Ocular Sarcoidosis; VKH: Vogt Koynagi Harada.

posterior synechiae and therefore was not evaluated. The mean central macular thickness (CMT) was $506.1 \mu \mathrm{m}(164-778 \mu \mathrm{m})$ (Table 4). During the first month follow-up, 12 eyes (85.7\%) presented with decrease of the CMT and two eyes (14.3\%) showed increase of the CMT. However, at 3-month follow-up the decrease of CMT was registered in only ten eyes (71.4\%). Four eyes (28.6\%) showed increase of CMT at this final follow-up. Change in the central macular thickness exceeding $100 \mu \mathrm{m}$ was considered significant, therefore $64.3 \%$ and $35.7 \%$ of the eyes studied showed improvement of CMT at 1 and 3-month follow-up, respectively. However, the CMT increased in $7.1 \%$ and $21.4 \%$ studied eyes, at the same follow-up interval.

\section{DISCUSSION}

In the current study, the most common underlying cause of $\mathrm{ME}$ was diabetic retinopathy, that can be explained by the high prevalence of diabetes mellitus worldwide. It is projected that by the year 2045 up to 700 million people will have diabetes ${ }^{4}$ and therefore it is mandatory to invest in a public health strategy that could prevent blindness due to diabetic macular edema.

Current treatment options for ME available in the Brazilian Health Public System are associated with significant limitations. The most used intravitreal anti-VEGF agent's regimens, pro re nata and treat and extend, include at least three loading doses of intravitreal drugs and further frequent medical appointments and monthly injections to manage the ME, becoming a burden to the patient and relatives ${ }^{7-9}$.

Our study showed that the medium number of previous intravitreal injections administered was seven per eye before the
Table 2: Initial visual acuity and in the first and third months.

\begin{tabular}{|c|c|c|c|}
\hline \multirow{2}{*}{ Patients } & \multicolumn{3}{|c|}{ Corrected visual acuity (decimal) } \\
\cline { 2 - 4 } & Initial & 1th month & 3th month \\
\hline 1 & 0.156 & 0.25 & 0.25 \\
\hline 2 & 0.08 & 0.16 & 0.16 \\
\hline 3 & 0.01 & 0.08 & 0.13 \\
\hline 4 & 0.05 & 0.08 & 0.13 \\
\hline 5 & 0.08 & 0.12 & 0.16 \\
\hline 6 & 0.25 & 0.12 & 0.12 \\
\hline 7 & 0.1 & 0.16 & 0.1 \\
\hline 8 & 0.2 & 0.2 & 0.2 \\
\hline 10 & 0.5 & 0.4 & 0.4 \\
\hline 11 & 0.25 & 0.08 & 0.03 \\
\hline 12 & 0.4 & 0.4 & 0.20 \\
\hline 13 & 0.05 & 0.13 & 0.08 \\
\hline 14 & 0.8 & 0.25 & 0.2 \\
\hline 15 & 0.05 & 0.12 & 0.05 \\
\hline
\end{tabular}

dexamethasone implant. The need to reduce the number of injections and invasive procedures and yet maintain de best visual acuity possible to the patient is a challenge to ophthalmologists.

In addition to their anti-VEGF property, corticosteroids, such as dexamethasone, suppress inflammation by inhibiting several inflammatory cytokines, resulting in decreased: edema, capillary leakage and migration of inflammatory cells. That is the reason why they should be considered as an alternative therapy to those patients presenting with chronic macular edema, that do not respond to exclusively anti-VEGF agent's intravitreal injections. 
Table 3: Measurements of intraocular pressure.

\begin{tabular}{|c|c|c|c|c|}
\hline \multirow{2}{*}{ Patients } & \multicolumn{5}{|c|}{ Intraocular pressure (mmHg) } \\
\cline { 2 - 5 } & Initial & 7th day & 1th month & 3th month \\
\hline 1 & 11 & 11 & 12 & 12 \\
\hline 2 & 16 & 12 & 13 & 13 \\
\hline 3 & 20 & 15 & 15 & 18 \\
\hline 4 & 11 & 15 & 18 & 18 \\
\hline 5 & 12 & 12 & 12 & 12 \\
\hline 6 & 18 & 18 & 17 & 17 \\
\hline 7 & 14 & 22 & 14 & 16 \\
\hline 8 & 9 & 9 & 9 & 9 \\
\hline 9 & 16 & 15 & 14 & 14 \\
\hline 10 & 15 & 9 & 18 & 10 \\
\hline 11 & 13 & 13 & 17 & 16 \\
\hline 12 & 18 & 18 & 22 & 22 \\
\hline 13 & 20 & 15 & 17 & 16 \\
\hline 15 & 16 & 17 & 28 & 14 \\
\hline & 20 & 28 & 12 & 16 \\
\hline
\end{tabular}

In our study, the favorable effect of dexamethasone implants was consistent with the one described in the literature and showed BCVA improvement and decreased central macular thickness measurement in most of the eyes during the first month of follow-up.

Limitations of our study include small sample size and short follow-up of only three months, which limits to access the risks of cataract formation and the effect of the elevated IOP in long term. However, the strength of this study was that we evaluated
Table 4: The mean central macular thickness.

\begin{tabular}{|c|c|c|c|}
\hline \multirow{2}{*}{ Patients } & \multicolumn{3}{|c|}{ Central macular thickness $(\boldsymbol{\mu m})$} \\
\hline & Initial & 1th month & 3th month \\
\hline 1 & 459 & 307 & 570 \\
\hline 2 & 548 & 280 & 511 \\
\hline 3 & 164 & 154 & 534 \\
\hline 4 & 490 & 254 & 247 \\
\hline 5 & 784 & 324 & 297 \\
\hline 6 & 609 & 486 & 476 \\
\hline 8 & 570 & 276 & 531 \\
\hline 9 & - & - & - \\
\hline 10 & 532 & 419 & 442 \\
\hline 11 & 428 & 499 & 643 \\
\hline 12 & 401 & 331 & 336 \\
\hline 13 & 360 & 333 & 335 \\
\hline 14 & 283 & 435 & 367 \\
\hline 15 & 778 & 245 & 235 \\
\hline & 680 & 314 & 260 \\
\hline
\end{tabular}

simultaneously the anatomical and visual acuity outcomes in $\mathrm{ME}$ secondary to different causes, mimicking the challenges faced in clinical practice.

In conclusion, the present study demonstrated that in patients with macular edema due to different underlying causes, a single of dexamethasone implant was well tolerated and produced improvements in visual acuity and central macular thickness. We aim to encourage the use of intravitreal dexamethasone implant as an alternative therapy for macular edema in Brazilian public health system.

\section{REFERENCES}

1. Flaxman SR, Bourne RRA, Resnikoff $S$, Ackland P, Braithwaite $\mathrm{T}$, Cicinelli MV, et al. Global causes of blindness and distance vision impairment 1990-2020: a systematic review and metaanalysis. Lancet Glob Health. 2017;5(12):e1221-34. https://doi.org/10.1016/S2214-109X(17)30393-5

2. Gupta N, Mansoor S, Sharma A, Sapkal A, Sheth J, Falatoonzadeh $\mathrm{P}$, et al. Diabetic retinopathy and VEGF. Open Ophthalmol J. 2013;7:4-10

https://doi.org/10.2174/1874364101307010004

3. Diabetic Retinopathy Clinical Research Network; Wells JA, Glassman AR, Ayala AR, Jampol LM, Aiello LP, et al. Aflibercept, bevacizumab, or ranibizumab for diabetic macular edema. N Engl J Med. 2015;372(13):1193-203

https://doi.org/10.1056/NEJMoa1414264

4. Bolinger MT, Antonetti DA. Antonetti. Moving past anti-VEGF: novel therapies for treating diabetic retinopathy. Int $\mathrm{J}$ Mol Sci. 2016;17(9):1498.

https://doi.org/10.3390/ijms17091498

5. Kanra AY, Akçakaya AA, Yaylalı SA, Altınel MG, Sevimli N. The efficacy and safety of intravitreal dexamethasone implant for the treatment of macular edema related to retinal vein occlusion: real- life data and prognostic factors in a Turkish population. Turk $J$ Ophthalmol. 2017;47(6):331-7.

https://doi.org/10.4274/tjo.75317

6. Lowder C, Belfort Jr R, Lightman S, Foster CS, Robinson MR, Schiffman $\mathrm{RM}$, et al. Dexamethasone intravitreal implant for noninfectious intermediate or posterior uveitis. Arch Ophthalmol. 2011;129(5):545-53. https://doi.org/10.1001/archophthalmol.2010.339

7. Chhablani J, Jhingan M, Goud A, Vupparaboina KK, Das T. Macular edema resolution assessment with implantable dexamethasone in diabetic retinopathy (MERIT): a pilot study. Clin Ophthalmol. 2018;12:1205-11.

https://doi.org/10.2147/OPTH.S163681

8. Demir G, Ozkaya A, Yuksel E, Erdogan G, Tunc U, Ocal MC, et al. Early and Late Switch from Ranibizumab to an intravitreal Dexamethasone implant in patients with Diabetic macular edema in the event of a poor anatomical response. Clin Drug Investig. 2020;40(2):119-28. https://doi.org/10.1007/s40261-019-00865-7

9. Hufendiek K, Pielen A, Framme C. Strategies of Intravitreal Injections with Anti-VEGF: "Pro re Nata versus Treat and Extend". Klin Monbl Augenheilkd. 2018;235(8):930-9. https://doi.org/10.1055/s-0042-122340 